\title{
Percepción de la actualización de la factura electrónica en los contribuyentes de la zona centro de La Barca (Jalisco)
}

\author{
Luz María Galán Briseño ${ }^{1}$, Miguel Castro Sánchez ${ }^{2}$ \\ y Alejandra Guadalupe Estrada Lomeli ${ }^{3}$ \\ ${ }^{1}$ Universidad de Guadalajara, Centro Universitario de la Ciénega, luz.galan@academicos.udg.mx, \\ Av. Universidad No. 1115 Colonia Linda Vista Ocotlán, Jalisco, México C. P. 47820, 3929400974. \\ ${ }^{2}$ Universidad de Guadalajara, Centro Universitario de la Ciénega, m.castro@academicos.udg.mx, \\ Av. Universidad No. 1115 Colonia Linda Vista Ocotlán, Jalisco, México C. P. $47820,3929400977$. \\ ${ }^{3}$ Universidad de Guadalajara, Centro Universitario de la Ciénega, alejandra.estrada@alumnos.udg.mx, \\ Av. Universidad No. 1115 Colonia Linda Vista Ocotlán, Jalisco, México C.P. 47820, 3931202918.
}

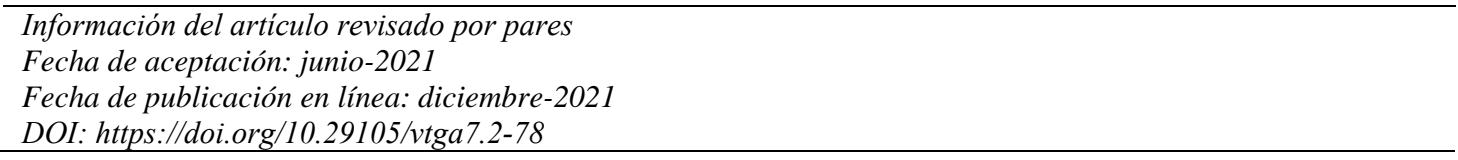

\section{Resumen}

La efectividad de la recaudación fiscal representa una de las acciones más importantes en la gestión y administración pública de los gobiernos para hacerse llegar de los recursos y así cumplir con los derechos constitucionales de la ciudadanía, es por ello que en los últimos años el Servicio de Administración Tributaria (SAT) ha implementado diversas reformas en materia de facturación electrónica mismas que últimamente han generado controversias en los contribuyentes para el cumplimiento efectivo de los requisitos en la elaboración del Comprobante Fiscal Digital por Internet (CFDI), el cual genera incertidumbre en el llenado de los apartados sustanciales que conforman la factura electrónica como la clave de producto o servicio, método de pago, tipo de comprobante y uso. El objeto del trabajo es analizar las nuevas imposiciones en materia de facturación electrónica y conocer su efecto en los contribuyentes, con el propósito de sugerir recomendaciones para atender sus necesidades y mitigar las circunstancias que obstaculizan la efectividad en el cumplimiento de las obligaciones fiscales. Para el propósito del trabajo se desarrolló una investigación mixta (cualitativa y cuantitativa), descriptiva y transversal, soportada

\section{Abstract}

The effectiveness of tax collection represents one of the most important actions in the management and public administration of governments to obtain resources and thus comply with the constitutional rights of citizens, that is why in recent years the Service of Tax Administration (SAT) has implemented various reforms in the field of electronic invoicing, which have lately generated controversies among taxpayers for the effective fulfillment of the requirements in the preparation of the Online Tax Receipt (CFDI), which generates uncertainty in the filling out of the substantial sections that make up the electronic invoice such as the product or service key, payment method, type of voucher and use. The purpose of the work is to analyze the new impositions in the matter of electronic invoicing and to know their effect on taxpayers, in order to suggest recommendations to meet their needs and mitigate the circumstances that hinder the effectiveness in complying with tax obligations. For the purpose of the work, a mixed (qualitative and quantitative), descriptive and cross-sectional investigation was developed, supported by the collection and analysis of data. The results show, on the part of the tax authority, lack of a timely 
por la recolección y análisis de datos. Los resultados muestran de parte de la autoridad impositiva, carencia de una política oportuna y efectiva de difusión de las reformas de facturación electrónica y que en el afán de dar respuesta a las obligaciones fiscales, provoca desinformación entre los contribuyentes, así mismo los obligados manifestaron carencia de sistemas de cómputo y dominio de tecnologías.

Palabras clave: factura electrónica, obligaciones fiscales, contribuyente, recaudación.

\section{TRODUCCIÓN}

El Servicio de Administración Tributaria (SAT), ha establecido criterios impositivos en el ejercicio de la facturación electrónica que buscan generar seguridad para los contribuyentes en la emisión de facturas de sus operaciones, criterios que los obligados requieren atender para dar cumplimiento en los términos de este ordenamiento de fiscalización tributaria, sin embargo, en este proceso el contribuyente manifiesta incertidumbre en cuanto a si es correcto el llenado de la factura electrónica en relación a los apartados que la componen como la clave de producto o servicio, método de pago, tipo de comprobante y uso de Comprobante Fiscal Digital por Internet (CFDI).

Como parte de la investigación, se describen los argumentos que dieron origen al interés de este trabajo, en este sentido es relevante analizar los cambios de esta fiscalización tributaria y en particular conocer la percepción sobre cuáles son las limitaciones y como son afectados los contribuyentes de la zona centro de La Barca, Jalisco durante el proceso de implementación de la facturación electrónica en sus negocios para dar respuesta a esta imposición de la autoridad.

Para este propósito resulta importante referir diversos trabajos que de manera análoga describen los efectos de este mandato tributario en el proceso de implementación en los negocios de los contribuyentes, en primer término, es de reconocerse que la facturación electrónica se encuentra alineada a la obtención y uso de las tecnologías de la información y comunicación, tal como lo describen De León et al. (2016), es necesario and effective policy for the dissemination of electronic invoicing reforms and that in the effort to respond to tax obligations, it causes misinformation among taxpayers, likewise the obliged ones expressed lack of computer systems and mastery of technologies.

Keywords: mental health, business, productivity.

JEL: H30, H32.

propiciar programas para la apropiación del uso de las tecnologías de información y comunicación mediante esquemas que promuevan la gestión del conocimiento en las MIPYMES, por lo que la exigencia de la facturación electrónica se convierte en una disrupción para dichas empresas en su carácter $y$ nivel de obligatoriedad que el SAT ha determinado.

La facturación electrónica ha generado muchas controversias ante los nuevos cambios, pues no se sabe con exactitud si se está cumpliendo con las expectativas del SAT, sobre todo cuando se trata de contribuyentes con poco conocimiento del tema, con lo son los pequeños empresarios dedicados a la realización de actividades comerciales, de servicios y manufactura, así como otros de menor presencia dedicados a la realización de actividades agrícolas y ganaderas, todos formando parte del municipio de La Barca, Jalisco, dentro del cual se respalda la investigación de campo del presente trabajo.

La incorporación de novedades en lo que a obligaciones fiscales se refiere, debe ser un proceso paulatino y apoyado con distintos recursos que solventen de forma oportuna las dudas que puedan ir surgiendo; adaptarse a los cambios asociados al uso de CFDI no es la excepción, pues continuamente se observa a los contribuyentes con escasa información de apoyo y que requieren estar continuamente informados y tener el apoyo de asesores expertos en el tema.

Aún y cuando se cuenta con distintas plataformas de apoyo en línea por parte del SAT, se siguen presentando cancelaciones de 
facturas por la no implementación correcta del procedimiento, en algunos casos se habla de hasta dos o tres por ciento de las mismas (Flores, 2018).

En una visión propositiva, los nuevos cambios propuestos al tema de factura electrónica buscan generar beneficios diversos, por un lado fortalecer los mecanismos de fiscalización del Servicio de Administración Tributaria (SAT) y por otro lado beneficios para el propio contribuyente, ya que permitirá hacer más eficiente el proceso para emitir sus CFDI y cumplir con sus obligaciones tributarias.

\section{MARCO TEÓRICO}

Con la intención de contar con los elementos suficientes para poder emitir recomendaciones, es momento de considerar la inclusión de los elementos teóricos de apoyo para el tema.

\section{1. ¿Qué es la factura electrónica?}

También conocida con el nombre de Comprobante Fiscal Digital por Internet (CFDI) es considerada por el Sistema de Administración Tributaria (SAT) como un documento de altos estándares de seguridad, y como lo refiere Aguilera (2010) se distingue por ser auténtico, íntegro, tónico y verificable, su validez es igual al de un comprobante fiscal impreso. Es auténtico porque permite verificar la identidad tanto del emisor como del receptor del documento; la integridad garantiza la seguridad de la información que incluye dicho documento; es verificable por la asignación que tiene de folios registrados, cadenas originales y sellos digitales. Es tónico porque tiene un folio, número de aprobación y vigencia certificada. Derivado de los distintos elementos de identificación y seguridad, el CFDI puede ser verificado para validar su autenticidad. Dicho documento electrónico puede integrar un número importante de elementos que fueron objeto de una venta, pero sólo dispone de un folio.

Atendiendo a su origen, el CFDI comprueba la transacción que involucra una serie de productos o servicios, siendo útil en los registros del emisor, receptor y terceros involucrados. La ventaja de dicho comprobante es su alta confiabilidad, garantizando la comprobación de operaciones en cualquiera que sea el hecho donde sea utilizada, desde el ámbito comercial y hasta el tributario.

Aún y cuando existe resistencia por parte de los contribuyentes en el manejo de documentos electrónicos, ésta característica busca facilitar su almacenamiento y transmisión, pues siempre se mantiene su originalidad y son garantía fehaciente de cada transacción.

Aún y cuando pueden existir similitudes entre los sistemas de facturación electrónica que existen en diferentes países, siempre existirán elementos clave que los diferenciarán, precisamente ahí estriba la originalidad y autenticidad de los mismos.

\subsection{Ventajas y desventajas de la factura electrónica.}

Desde la perspectiva de Zambrano (2018) la factura electrónica tiene una ventaja obvia por la gran cantidad de información que incluye, pero resalta beneficios adicionales asociados a la implementación de la misma que se alejan de obviedad; las ventajas se pueden concentrar en cuatro categorías: control tributario, dinámica económica, beneficios contables para contribuyentes y seguridad de la información. Además de que se consideran beneficios para los contribuyentes, también es cierto que el control tributario se ve fortalecido, así como la certeza en la realización de actividades económicas.

Ahora bien, la facturación electrónica de igual forma que otras herramientas digitales, tiene la necesidad de incorporar mejoras a su funcionamiento con el propósito de mantenerla vigente, en algunos casos se puede cuestionar tal procedimiento por la necesidad constante de actualización. Aún y cuando el SAT pone a disposición del contribuyente distintos espacios físicos o virtuales para su capacitación en el uso de dicha herramienta, siempre se cuestionarán los cambios continuos y se distinguirán como "desventajas", el rol que juegan los asesores fiscales en este proceso de actualización es fundamental. Para Martínez (2018) las mejoras o "actualizaciones" que implementa las autoridades con respecto a la factura 
electrónica, deben ser procedimientos normales y bien recibidos por los contribuyentes, pues la inversión que se hace redituará en beneficios de distinta índole.

Con el objeto de mostrar los beneficios que implica la actualización de la Factura electrónica, a continuación se muestran las adiciones que hizo el Sistema de Administración Tributaria (SAT, 2018) de la versión 3.2 (anterior) a la 3.3 (actual), considerando cada uno de los elementos incluidos.

Con respecto al elemento "catálogos" en la versión anterior (3.2) no existían, mientras que en la nueva se cuenta con un total de 17 nuevos catálogos, entre los que se encuentran: "aduana, clave producto/servicio, clave, unidad, código postal, forma de pago, impuesto, método de pago, moneda, número de pedimento aduana, país, patente aduanal, régimen fiscal, tasa o cuota, tipo de comprobante, tipo factor, tipo relación y uso CFDI". Con respecto a los beneficios mostrados por Zambrano (2018) las adiciones anteriores fortalecen las categorías de control tributario y seguridad de la información, pues se vuelve más analítica la identificación de la operación.

Con respecto al elemento "lugar de expedición", mientras que la versión anterior consideraba el domicilio completo, en la nueva versión se estandariza el domicilio usando el código postal del contribuyente y atendiendo al catálogo actualizado de códigos postales; como sucede con el elemento anterior, se fortalece la categoría de control tributario y ahora se precisa un beneficio contable para el contribuyente, pues se vuelve más práctico el registro de las operaciones.

El elemento "régimen fiscal" se fortalece en la nueva versión, pues ahora el registro corresponde al nuevo catálogo de régimen fiscal, mientras que en la versión anterior se podía omitir o anotar N/A; nuevamente se ven fortalecidas las categorías de control tributario, beneficio contable para el contribuyente y seguridad de la información. La omisión de un dato, como lo contemplaba la versión anterior, puede generar desinformación en el caso de que se quiera identificar una operación.
La "forma de pago" era un elemento que se encontraba invertido en la versión anterior, mientras que ahora se provocó un registro de acuerdo al nuevo catálogo de forma de pago. La seguridad en la información nuevamente fue la categoría de detonó un beneficio.

El "tipo de comprobante" fue un elemento que se complementó en la nueva versión, pues antes sólo existía: ingreso, egreso y traslado; ahora se añade el comprobante por nómina y pagos. La modificación anterior facilita el registro por parte del contribuyente pues le permite una identificación más clara de la operación.

Con respecto al "uso del CFDI" no existía la opción la opción de receptor, mientras que ahora el registro es conforme al catálogo de uso del CFDI. Se ampliaron las alternativas de uso para el contribuyente, lo cual facilitó la identificación de la operación.

El elemento "tipo de relación" no existía en la versión anterior, mientras que ahora es posible hacer el registro conforme a la relación que existe con otras facturas. Nuevamente se facilitó el registro del comprobante para el contribuyente.

El "CFDI relacionado" en la versión anterior no existía, pero ahora el registro se realiza conforme a los UIDD de las facturas relacionadas.

El elemento "descuento" en la versión anterior estaba presente pero sin especificación, ahora hay la posibilidad de registrar el descuento que sea aplicable a cada comprobante de operaciones.

Anteriormente la "clave producto o servicio" no existía, mientras que en la versión reciente se dio origen a un nuevo catálogo con diversas claves para identificar el producto $y / o$ servicio.

La "clave de unidad de medida" anteriormente no existía, pero ahora se registra de acuerdo al catálogo existente para tal fin; misma situación sucedió con el "impuesto aplicable", "tipo de factor" y "tasa cuota".

Nótese que las mejoras propuestas en la versión 3.3 de la factura electrónica, buscan fortalecer las cuatro categorías resaltadas por Zambrano (2018), generando seguridad, control y facilidad en el registro de 
operaciones. El reto para el contribuyente es capacitarse o buscar asesoría de personal especializado en el tema para que pueda aprovechar los esfuerzos que está haciendo la autoridad tributaria en la mejora de sus procesos de comprobación. Tal y como lo menciona Martínez (2018), la no atención de las actualizaciones anteriores, puede derivar en problemas de tipo contable, fiscal y administrativo.

Los cambios en cualquier procedimiento de registro manual y electrónico son necesarios y benéficos, Luna (2018) refiere que las constantes actualizaciones de la factura electrónica buscan facilitar la capacidad de la autoridad tributaria para hacerse de más información alusiva a las operaciones que realizan los contribuyentes.

En contraparte a los esfuerzos realizados por el SAT, y aun cuando los contribuyentes son conscientes de los beneficios que les provoca las actualizaciones, existen muchos de ellos que aún no se familiarizan con el proceso, Martínez (2018) destaca que todos se deben adaptar a dichos cambios y de ser necesario, invertir un mayor volumen de recursos para poder transitar totalmente a un escenario digital y con ello hacer nuestros los beneficios que esto implica.

Ahora bien, así como las actualizaciones de la factura electrónica confieren distintas ventajas como las mencionadas, en cierto momento, también se pueden hacer presentes algunas desventajas, como todo sistema de apoyo informático, entre las más comunes se encuentran las siguientes: riesgo de virus, daño en un componente de la computadora, actualizaciones costosas, programación del software de nuevos equipos adquiridos, limitantes para incorporar multiusuarios y riesgo de robo. Con el objeto de aminorar las desventajas mencionadas con anterioridad, los contribuyentes deben procurar contar con equipos con suficiente capacidad y características adecuadas al software que se utilizará, de lo contrario los resultados no serán óptimos; también se debe cuidar el mantener respaldos de la información, para evitar falta de eficiencia del sistema en caso de perder datos por mal funcionamiento, cortes de luz y ataques cibernéticos de mayor o menor impacto.

Es momento ahora de contrastar los fundamentos teóricos asociados a los beneficios que brinda la factura electrónica y sus actualizaciones, con respecto a lo que opinan los contribuyentes que serán objeto de estudio y que a fin de cuentas, son la principal masa crítica de contribuyentes que debe acatar tales medidas.

\section{MÉTODO}

La presente investigación es del tipo descriptiva-documental, se apoyará en el método cuantitativo pues se realizará una investigación de campo. Los sujetos de estudio se ubican en la zona centro del municipio de La Barca, Jalisco. La opinión de los contribuyentes se obtendrá mediante la técnica de encuesta, que tendrá como principal instrumento un cuestionario que consta de 15 ítems, en los cuales se combinó la posibilidad de obtener respuestas concretas con la opción de obtener respuestas abiertas y sobre todo aprovechas los deseos de los contribuyentes por expresarse.

La muestra de contribuyentes que fueron cuestionarios ascendió a 20 y representó una integración no probabilística y a conveniencia de la investigación; uno de los factores que influyó de manera importante en la integración de la muestra fueron las condiciones de distanciamiento generadas por la COVID-19, que aunque resulte muy redundante, ha influido de forma importante en la realización de investigaciones de campo. Se intentó ampliar la cobertura de la muestra aprovechando la comunicación electrónica, pero un número importante de contribuyentes no accedió a esa forma de contacto.

Previo a la presentación de resultados es importante mencionar algunas características de La Barca, Jalisco, municipio en el cual se identificaron los sujetos de estudio. Según el diagnóstico del municipio elaborado en Diciembre del 2019 (IIEG, 2019), la Barca forma parte de la región Ciénega (Ver Figura 1) y en el año 2010 contaba con 83 localidades, siendo su cabecera municipal la más poblada. La mayor presencia de actividades económicas se asocia 
al sector servicios $(41.96 \%)$ y el comercio (47.91\%), manufactura $(7.75 \%)$ y actividades agrícolas, transporte, construcción, minería, etc., en menor proporción. A noviembre del 2019 contaba con 3,444 unidades económicas.

Figura 1. Localización geográfica de La Barca, Jalisco.

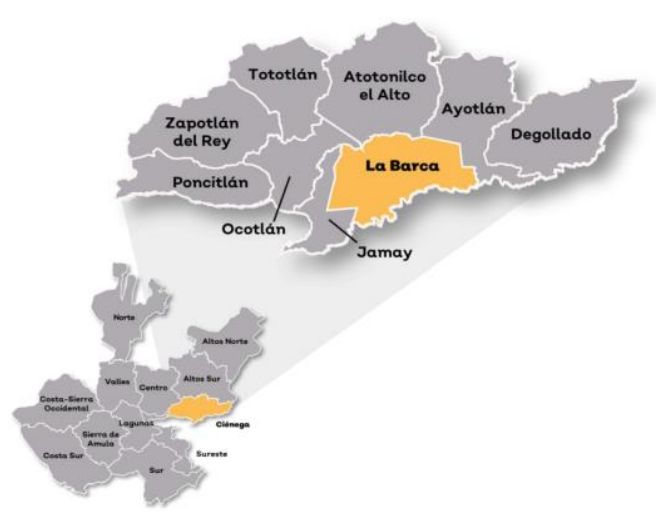

Fuente: Instituto de Información Estadística y Geográfica del Estado de Jalisco con base en "Mapa General del Estado de Jalisco, 2012”.

\section{RESULTADOS}

La precisión inicial que se hizo es el tipo de contribuyentes que será incluido en el estudio, resultando que $25 \%$ de ellos son personas morales y el resto personas físicas. Éste dato se consideró importante pues normalmente las empresas debidamente formalizadas tienen más apertura para las innovaciones tecnológicas que fortalecen sus procesos, precisamente lo que ocurre con el tema de las actualizaciones de la factura electrónica.

El siguiente dato que fue posible conocer de los sujetos de estudio, es su antigüedad como contribuyentes debidamente registrados, en la Tabla 1 se pueden observar los datos obtenidos.

Tabla 1. Antigüedad como contribuyente ante el Sistema de Administración Tributaria (SAT)

\begin{tabular}{cc}
\hline Categorías en años & $\%$ \\
\hline De 1 a 5 & 45 \\
De 6 a 10 & 5 \\
De 11 a 15 & 25 \\
De 16 a 20 & 5 \\
Más de 20 & 20 \\
\hline
\end{tabular}

Fuente: Investigación propia.
Mientras que cerca de la mitad de los contribuyentes son de reciente creación y pudieran catalogarse como micro y pequeñas empresas, el resto de los contribuyentes ya han podido experimentar cambios importantes en sus estructuras, sobre todo en lo que a cumplimiento de obligaciones tributarias se refiere.

La siguiente pregunta que hizo a los contribuyentes seleccionados buscó valorar sus conocimientos sobre la función del SAT y de alguna forma, la percepción que tienen de dicha autoridad, de manera concreta la pregunta fue ¿Qué es para usted el Servicio de Administración Tributaria (SAT)?, el concentrado de las respuestas obtenidas fue el siguiente: en el $60 \%$ de las respuestas predominó la consideración del SAT como una autoridad que se encarga de recaudar impuestos, mientras que el $40 \%$ restante lo visualiza como una institución que fomenta la contribución de las personas al gasto público, además de considerarla como una entidad ante la que se debe declarar los ingresos y gastos que se tienen. En general, la totalidad de los contribuyentes tiene claro que el SAT es un organismo encargado de la recaudación de impuestos, resultado del comparativo de ingresos y gastos que se tiene, para la realización de aportaciones significativas al gasto público.

En complemento a la pregunta anterior, se cuestionó lo siguiente: ¿Sabías que el Servicio de Administración Tributaria (SAT), es quien obliga y ayuda a que el contribuyente cumpla con sus obligaciones fiscales?; para este planteamiento no fue necesario elaborar ninguna gráfica o tabla, pues el 100\% respondió afirmativamente. Dicha respuesta era hasta cierto punto lógica, pues en la anterior la totalidad de los contribuyentes demostró amplio conocimiento al respecto.

Como pudo observarse en las dos preguntas anteriores, el conocimiento de las funciones del SAT es amplio pero ¿Conocen su página electrónica y los medios que ofrece para realizar la facturación electrónica?, el 65\% manifestó que si tiene conocimiento de dicha información, mientras que el 35\% tiene muy poco conocimiento al respecto. Haciendo 
una conexión con el primer ítem del cuestionario, se puede observar que el $50 \%$ de los contribuyentes que tiene mayor antiguiedad como contribuyente del SAT está familiarizado con el tema y seguramente ese $35 \%$ se encuentra entre los nuevos contribuyentes, particularmente aquellos cuya antigüedad no supera los 5 años. La adopción de la tecnología exige tiempo y al parecer la asociada al cumplimiento tributario exige más. En ese 35\% es donde el SAT debe tener particular atención, pues la falta de información se puede estar deformando hacia el incumplimiento de obligaciones y finalmente hacia la evasión fiscal.

La siguiente pregunta que se hizo a los contribuyentes fue ¿Qué tan favorable es para ti la página electrónica del SAT?, en éste ítem se manejaron varios niveles de respuesta que fueron del 1 (nada favorable) hasta 5 (ampliamente favorable), los resultados se pueden apreciar en la Tabla 2.

Nótese la aceptación que recibió la página del SAT y los servicios que ésta ofrece, pues $40 \%$ valoró como muy favorable la información que recibe, nuevamente se hicieron presentes los contribuyentes con mayor antigüedad, mientras que el resto de los contribuyentes eligieron las opciones 3 y 4 que van de medianamente favorable hasta favorable, lo cual es indicativo de que cuando ha existido necesidad de ingresar a la página electrónica correspondiente han resuelto en alguna medida sus dudas o dificultades.

Tabla 2. ¿Qué tan favorable es para ti la página del SAT?

\begin{tabular}{cc}
\hline Nivel de respuesta & $\%$ \\
\hline 1 & 0 \\
2 & 0 \\
3 & 20 \\
4 & 40 \\
5 & 40 \\
\hline
\end{tabular}

5. Fuente: Investigación propia.

Éste dato debe ser aliciente para las autoridades tributarias, pues el $100 \%$ de los contribuyentes ha ingresado a dicha plataforma, pues ninguno eligió la opción de nada favorable.

Independiente del tamaño y antigüedad del contribuyente, todos han tenido necesidad de utilizar la factura electrónica en cualquiera de sus actualizaciones, a ese respecto la siguiente pregunta que se hizo a los contribuyentes fue ¿Estás enterado de las nuevas actualizaciones de la factura electrónica?, para esta pregunta se manejaron 5 niveles de respuesta, donde 1 significa que no conoce información al respecto y 5 significaba que está ampliamente informado, las respuestas se pueden apreciar en la Tabla 3.

Tabla 3. ¿Estás enterado de las nuevas actualizaciones de la factura electrónica?

\begin{tabular}{cc}
\hline Niveles de respuesta & $\%$ \\
\hline 1 & 10 \\
2 & 30 \\
3 & 0 \\
4 & 0 \\
5 & 60 \\
\hline
\end{tabular}

Fuente: Investigación propia.

En ésta pregunta fue posible apreciar que aún y cuando los contribuyentes conocen ampliamente las funciones del Sistema de Administración Tributaria, así como los servicios que ofrece en su plataforma virtual, $40 \%$ aún desconoce de forma importante los lineamientos asociados a la factura electrónica y en particular los beneficios que se han obtenido con sus distintas actualizaciones. Teniendo presente que la factura electrónica es pieza clave para la comprobación de operaciones comerciales y principal medida de control tributario, no es un tema menor que deba ser descuidado. El SAT debe reforzar sus mecanismos de información y acercamiento al contribuyente, para capacitar a ese $40 \%$ de contribuyentes e impulsar su actualización con respecto al manejo de la factura electrónica.

Siguiendo con el tema de las actualizaciones de la factura electrónica, la siguiente pregunta que se hizo fue ¿De qué manera te enteraste de las nuevas actualizaciones?, en éste sentido el $75 \%$ de los contribuyentes manifestó que se enteró por medio de su asesor fiscal, mientras que el resto lo hizo por consultas realizadas en la página del SAT. En esta pregunta se puede 
visualizar la gran importancia que tienen los asesores quienes prácticamente son la voz del contribuyente en lo que al cumplimiento de sus obligaciones fiscales se refiere.

A continuación y en el mismo orden, se preguntó a los contribuyentes ¿Por qué crees que el SAT emite estas nuevas actualizaciones de la factura electrónica?, como se observó en la comparativa de la versión 3.2 y 3.3, el propósito de las actualizaciones es fortalecer los elementos asociados a la herramienta e incrementar el grado de identificación y seguridad de las operaciones del contribuyente. Las respuestas a la pregunta anterior, son congruentes con lo que plantean las actualizaciones, tal y como se puede apreciar en la Tabla 4.

Más del 50\% de los contribuyentes se enfocan en el beneficio de control tributario, resaltado por Zambrano (2018), mientras que el resto de los contribuyentes se inclinan por la opción de que facilita el cumplimento de las obligaciones tributarias.

Tabla 4. ¿Por qué crees que el SAT emite actualizaciones de la factura electrónica?

\begin{tabular}{cc}
\hline Opciones de respuesta & $\%$ \\
\hline Mayor control tributario & 55 \\
Incentivar el cumplimiento fiscal & 30 \\
Mejorar uso de las facturas & 10 \\
Eficiencia en la recaudación & 5 \\
\hline
\end{tabular}

Fuente: Investigación propia.

La siguiente pregunta estuvo dirigida hacia el grado de complejidad en el aprovechamiento de las actualizaciones que perciben los contribuyentes, las respuestas se pueden apreciar en la Tabla 5. En las categorías de respuesta que podían elegir los contribuyentes el 5 correspondía a muy fácil y el 1 correspondía a nada fácil.

Tabla 5. ¿Te ha sido fácil adaptarte a las actualizaciones de la factura electrónica?

\begin{tabular}{cc}
\hline Niveles de respuesta & $\%$ \\
\hline 1 & 5 \\
2 & 5 \\
3 & 50 \\
4 & 35 \\
5 & 5 \\
\hline
\end{tabular}

Fuente: Investigación propia.
Como se puede apreciar en la Tabla 5, para el 90\% de los contribuyentes la facilidad para implementar las actualizaciones ha estado en la escala de medianamente fácil hasta muy fácil y seguramente el rol de los asesores fiscales ha tenido mucho que ver y es congruentemente en gran medida con los resultados mostrados en la Tabla 3, donde el $60 \%$ de los contribuyentes está ampliamente enterado de las actualizaciones de la factura electrónica. Así como se resalta el papel que han desempeñado los asesores fiscales, también es de tomarse en cuenta el esfuerzo que ha realizado el SAT para fortalecer su plataforma electrónica y los recursos de apoyo al contribuyente que se encuentran en ella.

Con la intención de valorar la "holgura" en tiempo que brinda el SAT para adaptarse a las nuevas actualizaciones, la pregunta que se planteó fue la siguiente $¿ \mathrm{Te}$ parece suficiente el tiempo que concede el SAT a los contribuyentes para que se adapten a las nuevas actualizaciones?, los resultados que se pueden observar en la Tabla 6 muestran 5 categorías de respuesta, donde el 5 tiene que ver con total suficiencia y el 1 con total insuficiencia. La categoría de respuesta que más se hizo presente en opinión de los contribuyentes fue la de medianamente suficiente, quedando la máxima y menor categoría de respuesta.

Tabla 6. ¿Te parece suficiente el tiempo que concede el SAT a los contribuyentes para que se adapten a las nuevas actualizaciones?

\begin{tabular}{cc}
\hline Niveles de respuesta & $\%$ \\
\hline 1 & 0 \\
2 & 25 \\
3 & 45 \\
4 & 30 \\
5 & 0 \\
\hline Fuente: Investigación propia.
\end{tabular}

Los esfuerzos que está haciendo la autoridad tributaria están generando resultados favorables en cuando a la aceptación de las actualizaciones de la factura electrónica, pero aún falta trabajo por hacer en cuanto al tiempo que se concede a los contribuyentes para que se familiaricen con las modificaciones. 
La pregunta en este momento sería ¿Los contribuyentes disponen de los medios suficientes para cumplir con las nuevas actualizaciones de la factura electrónica?, a ese respecto, el $65 \%$ contestó positivamente, mientras que el resto manifestó que dispone de "muy pocos" medios para atender las actualizaciones. La justificación del mayor tiempo que demandan los contribuyentes para adoptar las actualizaciones de la factura electrónica, tiene relación con la disposición de medios para llevarlo a cabo.

En relación directa con los medios para atender las actualizaciones, se preguntó a los contribuyente ¿Qué sistema digital conoce y/o utiliza para la emisión de sus facturas electrónicas? el $52 \%$ está familiarizado con la página del SAT, mientras que el resto de los contribuyentes utiliza software especializado útil para la emisión de la factura. Ninguno de los contribuyentes manifestó desconocimiento de algún sistema relacionado.

La siguiente pregunta que se hizo fue si el contribuyente realiza sus propias facturas, debido al conocimiento de los medios digitales para ello. El $60 \%$ realiza dijo que ejecuta tal procedimiento de forma cotidiana $y$ atendiendo a sus propios medios, el resto necesita el apoyo de un asesor especializado para la emisión.

La pregunta de cierre que se hizo a los contribuyentes fue ¿Estás cumpliendo con las nuevas actualizaciones de la factura electrónica?, las respuestas se pueden observar en la Tabla 7. Los niveles de respuesta van del 1 al 5 , donde 5 expresa total cumplimiento y 1 nulo cumplimiento

Tabla 7. ¿Estás cumpliendo con las nuevas actualizaciones de la factura electrónica?

\begin{tabular}{cc}
\hline Niveles de respuesta & $\%$ \\
\hline 1 & 0 \\
2 & 0 \\
3 & 25 \\
4 & 60 \\
5 & 15 \\
\hline
\end{tabular}

Fuente: Investigación propia.

Sólo una menor proporción de los contribuyentes considera que está cumpliendo totalmente con las actualizaciones de la factura electrónica, mientras que el $85 \%$ está cumpliendo parcialmente. Tal y como se mostró con anterioridad, el cumplir con las actualizaciones de la factura electrónica, permite elevar el grado de control tributario y como se observa con esta pregunta, dicha situación no se está cumpliendo, pues existe un número importante de contribuyentes que por no contar con medios suficientes o falta de asesoría especializada, no está logrando el propósito.

Una vez concluido el análisis de los 15 ítems que incluyó el cuestionario para la obtención de datos, es momento de elaborar un resumen de las opiniones de los contribuyentes que tuvieron un nivel más alto de frecuencia y que será un preámbulo para la emisión de las conclusiones del trabajo.

El $75 \%$ de los sujetos de estudio fueron personas físicas, siendo minoría las opiniones que se obtuvieron de personas morales. En éste sentido las opiniones de los contribuyentes personas físicas pudieron verse afectadas por la falta de asesoría formal, a diferencia de las personas morales que normalmente tienen un departamento como para de su estructura que proporciona apoyo.

El $45 \%$ de las empresas objeto de estudio tenían una antigüedad como contribuyentes de 1 a 5 años, de igual forma éste dato puedo influir en la falta de madurez como organización y la necesidad de capacitación para cumplir con obligaciones fiscales.

El $60 \%$ de los contribuyentes visualiza al SAT como recaudador de impuestos, aún y cuando sea poco el conocimiento que se tenga de dicha institución, por el simple nombre ampliado se entienden que esa es su función.

La totalidad de los sujetos de estudio visualizan al SAT como un organismo que ayuda y obliga en el cumplimiento de obligaciones fiscales, éste dato es congruente con respecto a la forma como el $60 \%$ visualiza a dicha institución.

En cuanto al manejo de la plataforma electrónica del SAT, el $80 \%$ la valoró de forma favorable, señal de que en las ocasiones en que les ha tocado consultarla, su dificultad se ha resuelto. Sin duda las alternativas de apoyo en 
línea han provocado éste resultado, particularmente la opción de chatear en tiempo real y los números telefónicos para ampliar las consultas.

Con respecto a la factura electrónica y sus actualizaciones, el $60 \%$ de los contribuyentes dijo estar ampliamente enterado de dichos procesos. De forma adicional se puede mencionar, que también han sido objeto de las ventajas de dicho documento, sobre todo para otorgar seguridad en el registro de sus operaciones.

Tres cuartas partes de los contribuyentes manifestaron que la información asociada a las actualizaciones de la factura electrónica, la recibieron por parte del SAT y de su plataforma electrónica. El resto obtuvo la información de profesionales expertos en el tema.

El 55\% opina de forma favorable con respecto a que las actualizaciones de la factura electrónica son un gran apoyo como medio control tributario, inicialmente favorecen el trabajo del SAT pero también brindan seguridad a las operaciones realizadas por los contribuyentes.

$50 \%$ de los contribuyentes valora como fácil con sus limitantes, las actualizaciones de la factura electrónica, es decir, el acceso les parece adecuado a dicho instrumento de comprobación pero debería facilitarse aún más.

De los contribuyentes cuestionados, el $45 \%$ mencionó que el tiempo que concede el SAT para la adopción de las actualizaciones es medianamente aceptable, a criterio de dichos sujetos de estudio el tiempo asignado se debería ampliar, lo cual traería un mayor número de facilidades.

El $65 \%$ de los sujetos de estudio cuenta con los medios suficientes para las actualizaciones de la factura electrónica, el resto no cuenta con ellos y debe acudir a un asesor especializado para que lo apoye con los recursos tecnológicos necesarios.

El $60 \%$ de los contribuyentes elabora sus propias facturas electrónicas y ese mismo porcentaje manifestó estar cumpliendo de forma aceptable las nuevas actualizaciones.

En términos generales y aun cuando en su gran mayoría se trató de contribuyentes personas físicas, se tiene clara la función del Sistema de Administración Tributaria y un número importante acepta las ventajas de las actualizaciones en la factura electrónica.

\section{CONCLUSIONES}

La factura electrónica también conocida como CFDI es un documento que otorga al contribuyente una gran ventaja con respecto a la comprobación de sus operaciones, le otorga certeza en el registro de las mismas y brinda legalidad a las partes relacionadas.

También es cierto que el uso adecuado de los CFDI otorga una ventaja al Sistema de Administración Tributaria en términos de control impositivo, pues alimenta una base de datos que permite cuantificar las operaciones de una empresa y tener una base para la determinación de su base gravable.

El contar con una plataforma electrónica que apoya en la emisión de los CFDI sin duda representa una ventaja desde el punto de vista tecnológico, pues se trata de un sistema muy eficiente en cuanto al análisis y registro de datos $\mathrm{y}$, como toda herramienta tecnológica, requiere de actualizaciones constantes para poder procesar de manera eficiente los altos volúmenes de datos que se manejan. Así como el SAT hace esfuerzos por mantener en óptimas condiciones su plataforma, también los contribuyentes deben esforzarse en ese mismo sentido, haciendo suyas las actualizaciones correspondientes, por sus propios medios o con el apoyo de expertos en la materia.

Los servicios que proporciona el SAT son ampliamente identificados por los contribuyentes, valorando positivamente los servicios que les proporciona dicha institución. Cuando los sujetos de estudio tuvieron necesidad de ingresar a la plataforma de dicha institución normalmente los resultados fueron favorables.

Los recursos de apoyo que existen para la actualización del proceso de facturación electrónica se estar realizando de forma adecuada, lo que resta es que el SAT proporcione más holgura en los tiempos asignados a dichas actualizaciones. 


\section{REFERENCIAS}

Aguilera, G. (2010). Papelito habla y la factura electrónica también. https://search.ebscohost.com/login.aspx?direct=true\&db=a9h\&AN=54282148\&lang=es\&site=eh ost-live

De León, E. V., Cerón, L. M. T., León, M. F. J. \& Rodríguez, R. S. (2016). Impacto de la implementación de la factura electrónica en las MIPYMES del sector comercio y servicios en México. Revista Global de Negocios, 4(7), 85-94. En: https://www.theibfr.com/download/rgn/2016-rgn/rgn-v4n7-2016/RGN-V4N7-20168.pdf

Flores, L. (2018). Anuncian cambios en el procedimiento para cancelación de facturas. El Universal. https://amp.eluniversal.com.mx/amp/note/amp/eluniversal/1291535

Gobierno del Estado de Jalisco. (2019). H. Ayuntamiento de La Barca. https://www.jalisco.gob.mx/es/jalisco/municipios/la-barca

IIEG. (2019). La Barca, diagnóstico del municipio diciembre 2019. https://iieg.gob.mx/ns/wpcontent/uploads/2020/09/La-Barca-2.pdf

Luna, C. (2018). Factura electrónica del SAT: todo lo que debes saber. https://www.ccpm.org.mx/avisos/2018-2020/factura-electronica.pdf

Martínez, L. (2018). Impacto económico, tecnológico y administrativo que enfrentan las empresas al implementar el nuevo sistema de fiscalización del SAT. Universidad Autónoma de Aguascalientes. http://bdigital.dgse.uaa.mx:8080/xmlui/handle/11317/1608

SAT. (2018). ¿Qué obligaciones tiene un proveedor certificado de facturas. https://www.gob.mx/sat/acciones-y-programas/que-obligaciones-tiene-un-proveedor-decertificacion-de-factura

SAT. (s.f.). Cambios de la nueva factura electrónica 3.3 que debes conocer. https://satgobmx.com/cambios-la-nueva-factura-electronica-3-3-debes-conocer/ 